\title{
Uso de técnicas de regressão na avaliação, em bovinos de corte, da eficiência de conversão do alimento em produto: comparação entre grupos experimentais ${ }^{1}$
}

\section{Edenio Detmann², Sebastião de Campos Valadares Filho², Mateus Pies Gionbelli ${ }^{3}$, Pedro Veiga Rodrigues Paulino², Mário Fonseca Paulino²}

\footnotetext{
${ }^{1}$ Trabalho conduzido com apoio do CNPq, FAPEMIG (PPM) e INCT - Ciência Animal.

2 Departamento de Zootecnia, Universidade Federal de Viçosa, Viçosa-MG, 36571-000. Pesquisador do CNPq e do INCT-Ciência Animal.

${ }^{3}$ Programa de Pós-Graduação em Zootecnia, Universidade Federal de Viçosa.
}

RESUMO - Objetivou-se neste estudo propor e discutir um método de avaliação comparativa da eficiência de conversão do alimento em produto entre dois grupos experimentais de bovinos de corte baseado na utilização de técnicas de regressão Os procedimentos matemáticos e estatísticos foram desenvolvidos a partir de banco de dados formado pela mensuração do consumo de matéria seca e do ganho médio diário em 380 bovinos zebuínos puros ou mestiços em 15 experimentos constantes na base de dados do sistema BR-CORTE. Foram selecionados dois grupos constituídos por animais Nelore $(\mathrm{n}=156)$ e animais F1 Europeu $\times$ Nelore $(n=139)$. Foram propostas duas aproximações baseadas no ajustamento de modelos de regressão linear e não-linear, tendo o ganho médio diário com variável independente e o consumo de matéria seca como variável dependente. A utilização do alimento pelo animal foi estratificada em demanda para mantença e eficiência real de conversão em produto e as diferenças entre grupos foram avaliadas por intermédio de variáveis dummy. O critério de informação de Akaike foi proposto como ferramenta para escolha entre os modelos linear e não-linear. A avaliação comparativa da eficiência de transformação do alimento em produto entre dois grupos experimentais de bovinos de corte por meio de técnicas de regressão permite, caso pertinente, a estratificação dos grupos em função da eficiência de uso da dieta para suprimento de demandas para mantença e para produção.

Palavras-chave: critério de Akaike, regressão linear, regressão não-linear, variáveis dummy

\section{Use of regression techniques in the evaluation, in beef cattle, of feed conversion into product: comparison between experimental groups}

\begin{abstract}
The objective of this study was to propose and discuss a method to compare the feed into product conversion between two experimental groups of beef cattle based on regression techniques. The mathematical and statistical procedures were developed using a databank of dry matter intake and average daily gain of 380 purebred or crossbred zebu animals on the BR-CORTE nutritional system database. Two groups were selected from the databank and were formed by Nellore $(\mathrm{n}=156)$ or F1 European $\times$ Nellore $(n=139)$ animals. Two approaches were presented, based on linear and non-linear regression models fitting; the average daily gain and dry matter intake were used as independent and dependent variables, respectively. The food utilization by the animal was stratified into maintenance demand and true efficiency of product conversion. The difference between groups was evaluated by using dummy variables. The Akaike information criterion was suggested to decide which model is more adequate. The comparative evaluation of feed into product conversion between two experimental groups of beef cattle by using regression techniques allows, if suitable, the stratification of groups according to efficiency of diet utilization for maintenance and production.
\end{abstract}

Key Words: Akaike information criterion, dummy variables, linear regression, non-linear regression

\section{Introdução}

A eficiência de transformação do alimento em produto em avaliações de bovinos de corte é rotineiramente estimada pela razão entre o consumo voluntário de matéria seca (CMS) e o ganho médio diário (GMD), índice denominado conversão alimentar (CA). Contudo, diversas inconsistências estatísticas e nutricionais são apontadas sobre a conversão alimentar, o que pode comprometer a confiabilidade das inferências obtidas a partir desta (Guidoni, 1994; Detmann et al., 2005; Detmann et al., 2011).

Para contornar esses inconvenientes, tem sido proposta a avaliação simultânea dessas variáveis por intermédio da combinação linear baseada na técnica de variáveis 
canônicas, o que produz uma nova variável que preserva a característica de distribuição normal e engloba as propriedades discriminatórias das variáveis originais (Guidoni, 1994; Euclides Filho et al., 2002; Detmann et al., 2005). Esta nova variável, denominada índice bionutricional, possui, contudo, o inconveniente de seu valor ser dependente da estrutura da matriz residual dos dados do experimento avaliado (Detmann et al., 2005). Assim, tornamse impraticáveis a interpretação direta de seu significado numérico, a comparação entre estudos utilizando-se esse valor numérico e sua utilização como informação para programas de seleção e melhoramento genético animal (Detmann et al., 2011).

Consumo e ganho de peso são variáveis correlacionadas em um sistema de produção animal. A partir desta premissa, propôs-se recentemente a utilização de técnicas de regressão linear e não-linear para interpretação da eficiência de conversão do alimento em produto em bovinos de corte. No método proposto, a eficiência de determinado grupo de animais é avaliada por intermédio de dois parâmetros (demanda para mantença e eficiência real de conversão em produto), o que garante maior entendimento e confiabilidade às inferências nutricionais (Detmann et al., 2011).

No entanto, assumindo-se que comparações entre grupos são demandadas em diferentes situações experimentais e sistemas de produção, estabeleceu-se como objetivo neste estudo propor e discutir um método de comparação entre dois grupos experimentais de bovinos de corte quanto à eficiência de conversão do alimento em produto utilizando técnicas de regressão linear e não-linear.

\section{Material e Métodos}

Os procedimentos matemáticos e estatísticos foram desenvolvidos a partir de banco de dados formado pela mensuração do CMS e GMD em 380 bovinos zebuínos puros ou mestiços em 15 experimentos constantes na base de dados do sistema BR-CORTE (Valadares Filho et al., 2010). No conjunto de dados, foram avaliados animais machos não-castrados, machos castrados e fêmeas confinados com dieta à base de fenos (Cynodon sp. ou Brachiaria sp.), forragens pré-secadas (Cynodon sp. ou Brachiaria sp.), silagens (milho ou cana-de-açúcar) ou cana-de-açúcar in natura (Tabela 1; Figura 1).

Para ilustração dos procedimentos de comparação, foram selecionados dois grupos constituídos por animais Nelore $(n=156)$ e animais F1 Europeu $\times$ Nelore $(n=139)$ (Tabela 1; Figura 2).

A avaliação dos dados e os métodos propostos e discutidos foram desenvolvidos utilizando-se técnicas de regressão linear (Draper \& Smith, 1966; Myers, 1990) e não-linear (Bard, 1974; Souza, 1998). Os ajustamentos de modelos de regressão linear basearam-se no método dos mínimos quadrados ordinários e os de modelos de regressão

Tabela 1 - Caracterização quantitativa do banco de dados

\begin{tabular}{|c|c|c|c|}
\hline \multirow[t]{2}{*}{ Item } & \multicolumn{3}{|c|}{ Variável } \\
\hline & Ganho médio diário (kg/dia) & Consumo de matéria seca (kg/dia) & Conversão alimentar $(\mathrm{kg} / \mathrm{kg})$ \\
\hline & \multicolumn{3}{|c|}{ Banco de dados total } \\
\hline Mediana & 1,08 & 7,75 & 7,35 \\
\hline Moda & 1,21 & 8,22 & - \\
\hline Máximo & 1,97 & 12,53 & 21,22 \\
\hline \multirow[t]{2}{*}{$\mathrm{n}$} & \multicolumn{3}{|c|}{380} \\
\hline & \multicolumn{3}{|c|}{ Nelore } \\
\hline Média & 0,95 & 7,08 & 7,99 \\
\hline Mediana & 0,93 & 7,28 & 7,23 \\
\hline Moda & 0,97 & 7,33 & - \\
\hline Máximo & 1,71 & 9,74 & 20,89 \\
\hline Média & 1,17 & 8,37 & 7,36 \\
\hline Mediana & 1,20 & 8,17 & 7,15 \\
\hline Moda & 1,24 & 6,23 & 6,02 \\
\hline Máximo & 1,93 & 12,53 & 13,61 \\
\hline Mínimo & 0,61 & 5,49 & 4,81 \\
\hline s & 0,27 & 1,47 & 1,48 \\
\hline $\mathrm{n}$ & & 139 & \\
\hline
\end{tabular}




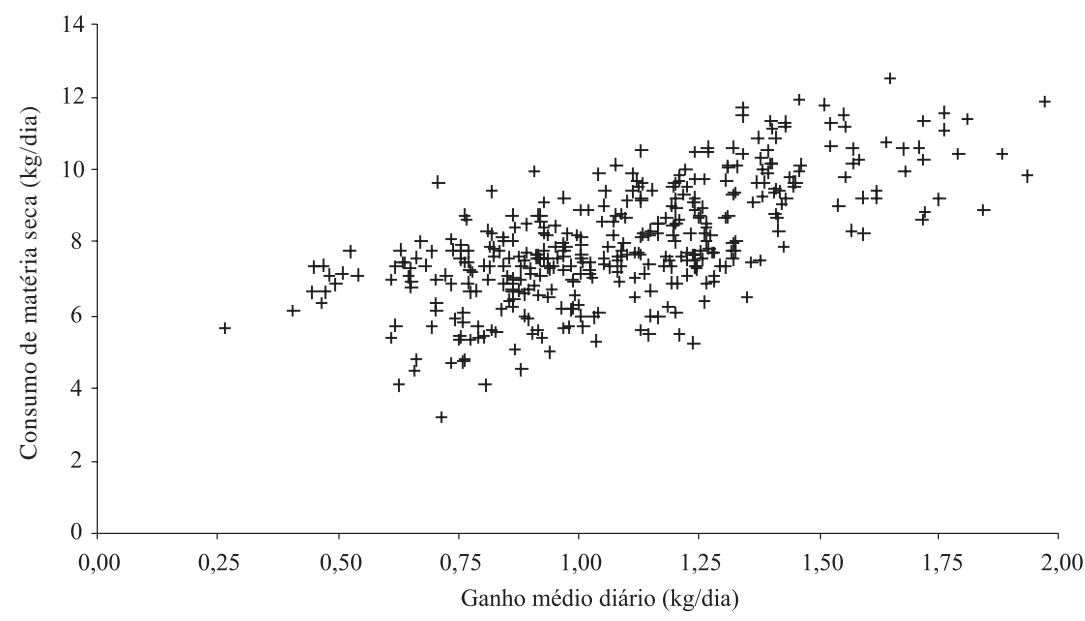

Figura 1 - Dispersão do consumo de matéria seca em função do ganho médio diário para o conjunto total de dados (n = 380).

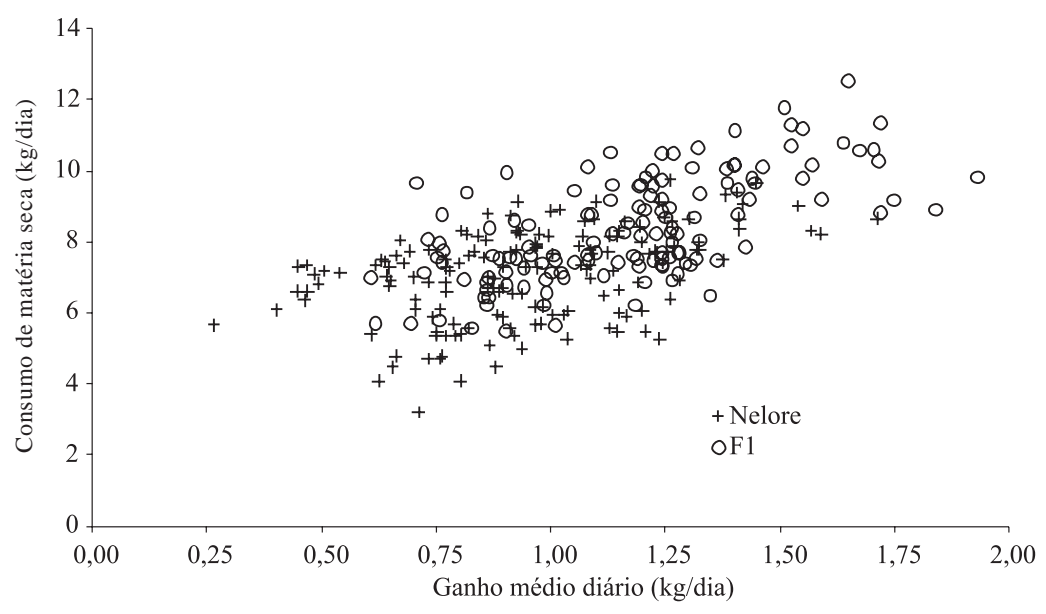

Figura 2 - Dispersão do consumo de matéria seca em função do ganho médio diário considerando-se animais Nelore (n=156) e F1 Europeu $\times$ Nelore $(n=139)$.

não-linear basearam-se no método de mínimos quadrados não-lineares com o uso do algoritmo iterativo de GaussNewton. Os métodos de comparação propostos foram baseados na utilização de variáveis dummy, tanto no caso linear (Draper \& Smith, 1966) como não-linear (Regazzi, 2003; Regazzi \& Silva, 2004, 2010). As análises foram conduzidas por intermédio do programa SAS ${ }^{1}$ (PROC GLM ePROC NLIN).

Na avaliação da eficiência de conversão do alimento em produto com base em técnicas de regressão, considerase que o CMS constitui a variável-resposta a ser investigada no sistema biológico representado pelo animal em produção e que ao menos parte da explicação do comportamento desta variável possa ser atribuída ao nível de produção (GMD).
Considerando-se modelo linear nos parâmetros, a relação entre CMS e GMD é descrita por (Detmann et al., 2011): $C \hat{M} S=f(X) \Rightarrow f(X)=\hat{Y} \Rightarrow \hat{Y}=\hat{\beta}_{0}+\hat{\beta}_{1} \times X$ em que: $\mathrm{X}$ = ganho médio diário; e $\mathrm{Y}=$ consumo voluntário de matéria seca.

Com a utilização de técnicas de regressão, pressupõese que a eficiência de conversão do alimento em produto seja definida por dois parâmetros: demanda para mantença (DM) e eficiência real de conversão em produto (ERCP).

Define-se como DM a porção da matéria seca total ingerida que é necessária para manutenção das funções basais do animal sem que haja mobilização de reservas (perda de peso) ou construção de produto (ganho de peso). Dessa forma, DM representa a porção do alimento ingerido que efetivamente não pode ser contabilizada como

${ }^{1}$ Statistical Analysis System, versão 9.1, licenciado para a Universidade Federal de Viçosa. 
substrato para síntese de produto (Detmann et al., 2011). Por definição, a DM é obtida sob condição de variação de peso corporal nula, implicando na definição matemática:

$D M_{L}=\lim _{X \rightarrow 0} f(X)=\hat{\beta}_{0}$

em que $\mathrm{DM}_{\mathrm{L}}$ é a demanda para mantença para o caso de utilização de modelo linear.

Por outro lado, define-se como ERCP a eficiência de conversão do alimento em produto obtida após o suprimento das demandas para mantença. Assim, ERCP denota a eficiência de produção a partir da porção teórica do alimento ingerido que efetivamente pode ser considerada para síntese de tecidos. Matematicamente a estimativa de ERCP para o caso do modelo linear é dada por (Detmann et al., 2011):

$E R C P_{L}=\frac{d f(X)}{d X}=\frac{d \hat{Y}}{d X}=\hat{\beta}_{1}$

em que $\mathrm{ERCP}_{\mathrm{L}}$ é a eficiência real de conversão em produto para o caso de utilização de modelo linear.

Para manutenção das coerências biológica e nutricional na descrição da relação entre GMD e CMS por intermédio do modelo linear as seguintes restrições devem ser observadas (Detmann et al., 2011):

$D M>0$

$E R C P>0$

As restrições indicam que em toda e qualquer situação produtiva sempre haverá necessidade de se suprirem demandas de mantença do animal (4a) e que qualquer substrato capaz de fornecer nutrientes e atributos nutricionais ao animal pode ser potencialmente utilizado para síntese de produto (4b) depois de supridas as demandas para mantença.

Em situações em que a relação entre GMD e CMS envolve a comparação de dois grupos, demanda-se a introdução de variável categórica no modelo linear originalmente proposto (Equação 1). Neste caso, níveis são atribuídos a esta variável de forma a se considerar o fato de que diferentes grupos podem apresentar efeitos diferentes sobre a variável-resposta (Draper \& Smith, 1966). Assim, a variável categórica "grupos" é introduzida no modelo na forma de variável dummy ou variável indicadora, gerando a estrutura de modelo ajustado:

$C \hat{M} S=f(X, D) \Rightarrow f(X, D)=\hat{Y} \Rightarrow \hat{Y}=\hat{\beta}_{0}+\hat{\beta}_{1} \times D+\hat{\beta}_{2} \times X+$

$+\hat{\beta}_{3} \times(X \times D)$

em que: $\mathrm{X}=$ ganho médio diário; $\mathrm{Y}=$ consumo voluntário de matéria seca; e $\mathrm{D}$ = variável dummy ou variável indicadora.

Para comparação entre dois grupos hipotéticos I e II, D assumirá valor zero para o grupo I e valor um para o grupo II. Sob estes pressupostos, os parâmetros $\beta_{1}$ e $\beta_{3}$ propiciam a comparação direta entre os grupos, uma vez que considerarão somente os valores oriundos do grupo II. Assim, para o caso de significância destes, diferenças entre grupos serão determinadas quanto ao intercepto e ao coeficiente de inclinação da função, respectivamente.

Considerando os parâmetros biológicos DM e ERCP, para o caso de haver significância quanto aos parâmetros $\beta_{1}$ e $\beta_{3}$ descritos em (5), tem-se:

$D M_{L}=\lim _{X \rightarrow 0} f(X, D)=\hat{\beta}_{0}+\hat{\beta}_{1} \times D$

$D M_{L}=\lim _{X \rightarrow 0} f(X \mid D=0)=\hat{\beta}_{0}$

$D M_{L}=\lim _{X \rightarrow 0} f(X \mid D=1)=\hat{\beta}_{0}+\hat{\beta}_{1}$

$E R C P_{L}=\frac{\partial f(X, D)}{\partial X}=\frac{\partial \hat{Y}}{\partial X}=\hat{\beta}_{2}+\hat{\beta}_{3} \times D$

$E R C P_{L}=\frac{\partial f(X \mid D=0)}{\partial X}=\frac{\partial \hat{Y}}{\partial X}=\hat{\beta}_{2}$

$E R C P_{L}=\frac{\partial f(X \mid D=1)}{\partial X}=\frac{\partial \hat{Y}}{\partial X}=\hat{\beta}_{2}+\hat{\beta}_{3}$

Não havendo significância quanto aos parâmetros $\beta_{1}$ e $\beta_{3}$ (Equação 5), DM e ERCP serão descritas por intermédio das estimativas dos parâmetros $\beta_{0}$ e $\beta_{2}$ para ambos os grupos experimentais em comparação, respectivamente (Equações 6 e 7).

Em algumas situações, a suposição de eficiência fixa e independente do nível de produção tem sido questionada com base no fato de haver redução na eficiência de conversão do alimento em produto à medida em que se amplia a intensidade do processo produtivo (Lana et al., 2005; Detmann et al., 2011). Desta forma, a eficiência seria definida como uma função do nível de produção, caracterizando função não-linear em seus parâmetros, a qual é descrita por:

$C \hat{M S}=f(X) \Rightarrow f(X)=\hat{Y} \Rightarrow \hat{Y}=\hat{\theta}_{0} \times e^{\hat{\theta}_{1} \times X}$ em que: $\mathrm{X}=$ ganho médio diário; e $\mathrm{Y}=$ consumo voluntário de matéria seca.

Utilizando-se o conceito de partição de eficiência apresentado anteriormente, estimam-se DM e ERCP na solução não-linear como:

$D M_{N L}=\lim _{X \rightarrow 0} f(X)=\hat{\theta}_{0}$

$E R C P_{N L}=\frac{d f(X)}{d X}=\frac{d \hat{Y}}{d X}=\hat{\theta}_{1} \times \hat{\theta}_{0} \times e^{\hat{\theta}_{1} \times X}$

em que: $\mathrm{DM}_{\mathrm{NL}}=$ demanda para mantença para o caso de utilização de modelo não-linear; e $\mathrm{ERCP}_{\mathrm{NL}}=$ eficiência real de conversão em produto para o caso de utilização de modelo não-linear.

Sob os mesmos argumentos apresentados para a equação (4), impõem-se as restrições à aplicação do modelo não-linear:

$D M>0 \Leftrightarrow \hat{\theta}_{0}>0$

$E R C P>0 \Leftrightarrow \hat{\theta}_{0}>0$ e $\hat{\theta}_{1}>0$ 
Para comparação entre dois grupos quanto à relação entre GMD e CMS, procede-se à reparametrização do modelo descrito em (10) considerando-se a utilização de duas diferentes variáveis dummy (Regazzi, 2003; Regazzi \& Silva, 2004; 2010), o que produz a estrutura de modelo ajustado: $C \hat{M} S_{\Omega}=D_{1} \times\left[\hat{\theta}_{01} \times e^{\hat{\theta}_{11} \times X}\right]+D_{2} \times\left[\hat{\theta}_{02} \times e^{\hat{\theta}_{12} \times X}\right]$ em que: $C \hat{M} S_{\Omega}=$ consumo estimado de matéria seca considerando-se que nenhuma restrição seja considerada quanto aos parâmetros $\theta_{0}$ e $\theta_{1}$; e $\mathrm{D}_{1}$ e $\mathrm{D}_{2}=$ variáveis dummy ou variáveis indicadoras.

Para comparação entre dois grupos hipotéticos I e II, $\mathrm{D}_{1}$ assumirá valor zero para o grupo I e valor um para o grupo II, ao passo que $\mathrm{D}_{2}$ assumirá valor um para o grupo I e valor zero para o grupo II (Equação 12).

Quando considerada a comparação entre dois grupos utilizando-se modelo linear, uma única variável dummy é utilizada, a qual permite estimar diretamente a diferença entre grupos quanto aos parâmetros DM e ERCP (Equação 5). Contudo, o modelo não-linear (Equação 15) apresenta estrutura que não permite a utilização de uma variável dummy como no caso linear. Desta forma, duas variáveis dummy são demandadas, as quais apresentam função de exclusão das observações de um grupo quando o comportamento de outro está sendo considerado.

O procedimento de comparação por intermédio do modelo não-linear se baseia na utilização de teste de razão de máxima verossimilhança (Regazzi, 2003). Este procedimento se inicia com o ajustamento de modelo considerando-se que os dois parâmetros não lineares são diferentes para os grupos em comparação, o que equivale ao ajustamento do modelo descrito na equação (12).

Após o ajustamento do modelo completo, no qual os grupos são assumidos como diferentes em ambos os parâmetros, dois novos ajustamentos devem ser realizados, nos quais são consideradas restrições sobre cada um dos parâmetros originais (Tabela 4), ou seja, considerando-se que os grupos são similares, resultando em:

$$
\begin{aligned}
& C \hat{M} S_{W}=D_{1} \times\left[\hat{\theta}_{0} \times e^{\hat{\theta}_{11} \times X}\right]+D_{2} \times\left[\hat{\theta}_{0} \times e^{\hat{\theta}_{12} \times X}\right] \\
& C \hat{M} S_{W}=D_{1} \times\left[\hat{\theta}_{01} \times e^{\hat{\theta}_{1} \times X}\right]+D_{2} \times\left[\hat{\theta}_{02} \times e^{\hat{\theta}_{1} \times X}\right]
\end{aligned}
$$

em que: $C \hat{M} S_{W}=$ consumo estimado de matéria seca considerando-se restrição quanto aos parâmetros $\theta_{0}$ e $\theta_{1}$ nas equações (13) e (14), respectivamente.

A partir das informações geradas pelo ajustamento dos três diferentes modelos (Equações 12, 13 e 14), testes de razão de máxima verossimilhança são executados para cada um dos parâmetros $\left(\theta_{0}\right.$ e $\left.\theta_{1}\right)$ utilizando-se a distribuição de $\chi^{2}$, sendo o valor calculado da estatística e os graus de liberdade dados por (Regazzi, 2003):
$\chi_{C A L C}^{2}=-n \times \ln \left(\frac{S Q R_{\Omega}}{S Q R_{W}}\right)$

$v=p(\Omega)-p(W)$

em que: $\mathrm{SQR}_{\Omega}=$ soma de quadrado residual do modelo ajustado sem restrições (Equação 12); $\mathrm{SQR}_{\mathrm{W}}=$ soma de quadro residual do modelo ajustado com restrição (Equação 13 ou 14); n = número de observações; $v$ = graus de liberdade; e p $(\Omega)$ e p $(W)$ = número de parâmetros dos modelos ajustados sem e com restrição, respectivamente.

Diferentemente do caso linear, as hipóteses testadas com a avaliação via modelo não-linear não podem ser interpretadas diretamente devido à interrelação dos parâmetros na definição das características biológicas (Equações 9, 10 e 11).

A despeito da maior complexidade do significado das hipóteses associadas ao modelo não-linear, considerandose que ambos os parâmetros sejam considerados diferentes entre grupos, as estimativas de DM e ERCP serão dadas por: $D M_{N L}=\lim _{X \rightarrow 0} f\left(X, D_{1}, D_{2}\right)=D_{1} \times \hat{\theta}_{01}+D_{2} \times \hat{\theta}_{02}$

$D M_{N L}=\lim _{X \rightarrow 0} f\left(X \mid D_{1}=1 ; D_{2}=0\right)=\hat{\theta}_{01}$

$D M_{N L}=\lim _{X \rightarrow 0} f\left(X \mid D_{1}=0 ; D_{2}=1\right)=\hat{\theta}_{02}$

$E R C P_{N L}=\frac{\partial f\left(X, D_{1}, D_{2}\right)}{\partial X}=\frac{\partial \hat{Y}}{\partial X}=D_{1} \times\left[\hat{\theta}_{11} \times \hat{\theta}_{01} \times e^{\hat{\theta}_{11} \times X}\right]+$

$+D_{2} \times\left[\hat{\theta}_{12} \times \hat{\theta}_{02} \times e^{\hat{\theta}_{12} \times X}\right]$

$E R C P_{N L}=\frac{\partial f\left(X \mid D_{1}=1 ; D_{2}=0\right)}{\partial X}=\frac{\partial \hat{Y}}{\partial X}=\hat{\theta}_{11} \times \hat{\theta}_{01} \times e^{\hat{\theta}_{11} \times X}$

$E R C P_{N L}=\frac{\partial f\left(X \mid D_{1}=0 ; D_{2}=1\right)}{\partial X}=\frac{\partial \hat{Y}}{\partial X}=\hat{\theta}_{12} \times \hat{\theta}_{02} \times e^{\hat{\theta}_{12} \times X}$

Em função de diferenças no tocante às aproximações estatísticas aplicadas a modelos lineares e não-lineares, a variância da relação entre GMD e CMS deve ser estimada pelo método da máxima verossimilhança, cujo estimador é definido por:

$s_{X Y}^{2}=\frac{S Q R}{n}=\frac{\sum_{i=1}^{n} e_{i}^{2}}{n}=\frac{\sum_{i=1}^{n}\left(Y_{i}-\hat{Y}_{i}\right)^{2}}{n}$

$s_{X Y}=\sqrt{s_{X Y}^{2}}$

A partir da estimativa de máxima verossimilhança da variância da relação entre GMD e CMS, sugere-se o cômputo do critério de informação de Akaike (AIC), que constitui procedimento relativo para identificação do melhor modelo de ajustamento aos dados, o qual pondera para o número de parâmetros e busca tornar a avaliação isenta de ambiguidades associadas a testes de hipóteses convencionais (Akaike, 1974).

Para o caso de modelos de regressão, o AIC pode ser estimado por (Kaps \& Lamberson, 2004):

$A I C=n \times \ln \left(s_{X Y}^{2}\right)+2 \times p$ 
em que p representa o número de parâmetros ajustados independentemente no modelo.

\section{Resultados e Discussão}

Para ilustração do procedimento comparativo utilizandose modelo linear, assumiu-se valor zero para a variável dummy para animais Nelore $(\mathrm{D}=0)$ e valor um para animais F1 Europeu $\times$ Nelore $(\mathrm{D}=1)$.

Segundo os métodos aqui propostos, o procedimento de comparação se inicia com o ajustamento de modelo considerando-se todos os possíveis parâmetros (Equação 5), cujo programa aplicado ao software SAS é apresentado na Tabela 2 (linhas 300 a 302). Após o ajustamento, devem ser observados os níveis descritivos de probabilidade para o erro tipo I (Valor-P) dos parâmetros associados à variável indicadora $\left(\beta_{1}\right.$ e $\left.\beta_{3}\right)$. Verificando-se significância para ambos, o modelo ajustado é adotado na descrição da relação entre CMS e GMD. Caso contrário, o(s) parâmetro(s) $\left(\beta_{1}\right.$ e $\beta_{3}$ ) que não apresenta(m) significância deve(m) ser omitido(s) do modelo e um novo ajustamento deve ser realizado.

Para o caso específico da comparação entre animais Nelore e F1 Europeu × Nelore, não se observou significância
( $\mathrm{P}>0,05)$ quanto ao parâmetro $\beta_{1}$ (Tabela 3). A partir da omissão deste do modelo, procedeu-se a novo ajustamento (Tabela 2, linhas 303 a 305), obtendo-se:

$C \hat{M} S=4,795( \pm 0,268)+2,382( \pm 0,277) \times G M D+0,689( \pm 0,131) \times$

$\times(G M D \times D) \quad\left(\mathrm{s}_{\mathrm{XY}}=1,144\right)$

em que: $\mathrm{D}=0$ para animais Nelore; e $\mathrm{D}=1$ para animais F1 Europeu $\times$ Nelore.

A ausência de significância $(P>0,05)$ para $\beta_{1}$, associada à significância $(\mathrm{P}<0,05)$ para $\beta_{3}$ (Tabela 3$)$, indica que os grupos apresentam DM similares, mas ERCP diferentes (Figuras 3 e 4). Neste contexto, aplicando-se as definições matemáticas anteriormente apresentadas, tem-se:

$$
\begin{aligned}
& D M_{L}=\lim _{X \rightarrow 0} f(X, D)=4,795 \\
& E R C P_{L}=\frac{\partial f(X \mid D=0)}{\partial X}=\frac{\partial \hat{Y}}{\partial X}=2,382 \\
& E R C P_{L}=\frac{\partial f(X \mid D=1)}{\partial X}=\frac{\partial \hat{Y}}{\partial X}=2,383+0,689=3,071
\end{aligned}
$$

A DM para ambos os grupos é estimada em 4,795 kg MS/dia (Equação 23; Figura 3). Por outro lado, a avaliação de ERCP indicou maior eficiência para animais Nelore, por demandarem menor consumo para produção $(2,382 \mathrm{~kg}$ MS/kg GMD) em comparação aos animais F1 Europeu × Nelore (3,071 kg MS/kg GMD) (Equação 24; Figura 4). Ressalta-se, contudo, que este resultado deve ser entendido

Tabela 2 - Programa utilizado no SAS para comparação entre grupos de acordo com o modelo linear (Equações 5 e 22)

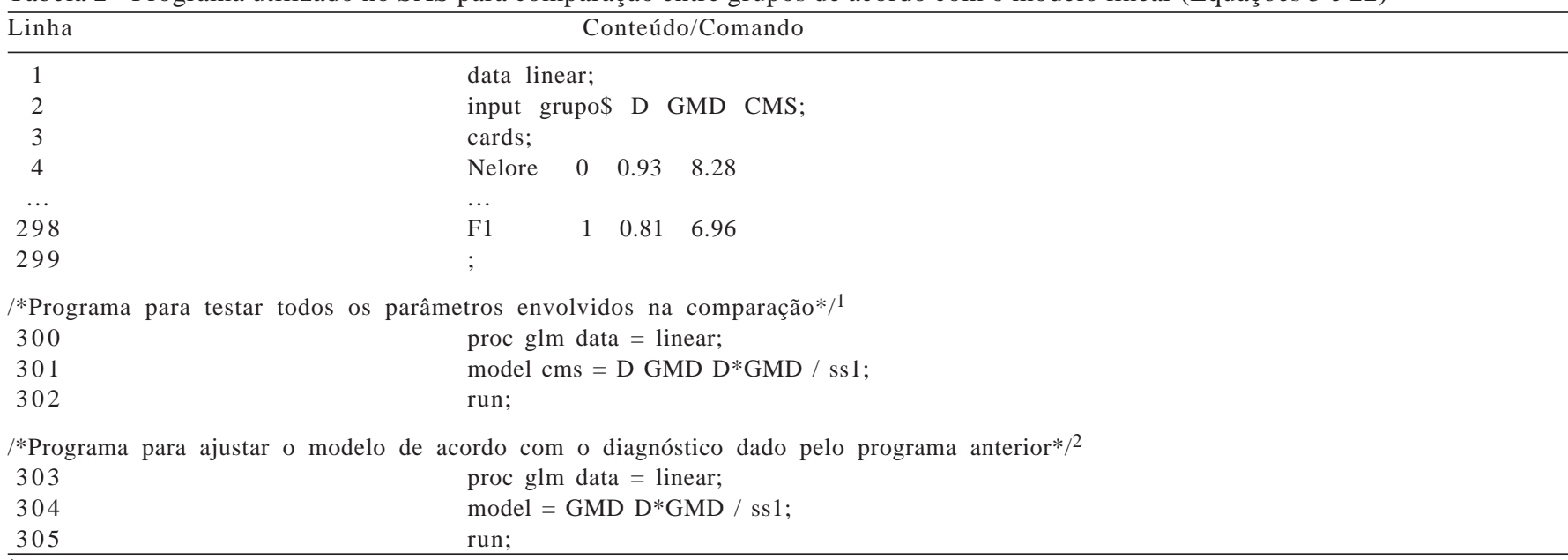

${ }^{1}$ Verificar Equação 5.

2 Para maiores detalhes, consultar a Tabela 3.

\begin{tabular}{|c|c|c|c|}
\hline \multirow[b]{2}{*}{ Item } & \multicolumn{2}{|c|}{ Hipóteses ${ }^{1}$} & \multirow[b]{2}{*}{ Valor-P } \\
\hline & Nulidade & Alternativa & \\
\hline Intercepto $\left(\beta_{0}\right)$ & $\mathrm{DM}_{1}=0$ & $\mathrm{DM}_{1} \neq 0$ & $<0,0001$ \\
\hline $\operatorname{GMD}\left(\beta_{2}\right)$ & $\mathrm{ERCP}_{1}=0$ & $\mathrm{ERCP}_{1} \neq 0$ & $<0,0001$ \\
\hline $\mathrm{GMD} \times \mathrm{D}\left(\beta_{3}\right)^{2}$ & $\mathrm{ERCP}_{1}=\mathrm{ERCP}_{2}$ & $\mathrm{ERCP}_{1} \neq \mathrm{ERCP}_{2}$ & 0,0094 \\
\hline
\end{tabular}

Tabela 3 - Interpretação das hipóteses estatísticas e nível descritivo de probabilidade para o erro tipo I (Valor-P) para o modelo linear (Equações 5 e 22)

${ }^{1}$ Grupo 1 = Nelore; Grupo 2 = F1.

$2 \mathrm{D}=0$ para Nelore; $\mathrm{D}=1$ para $\mathrm{F} 1$. 


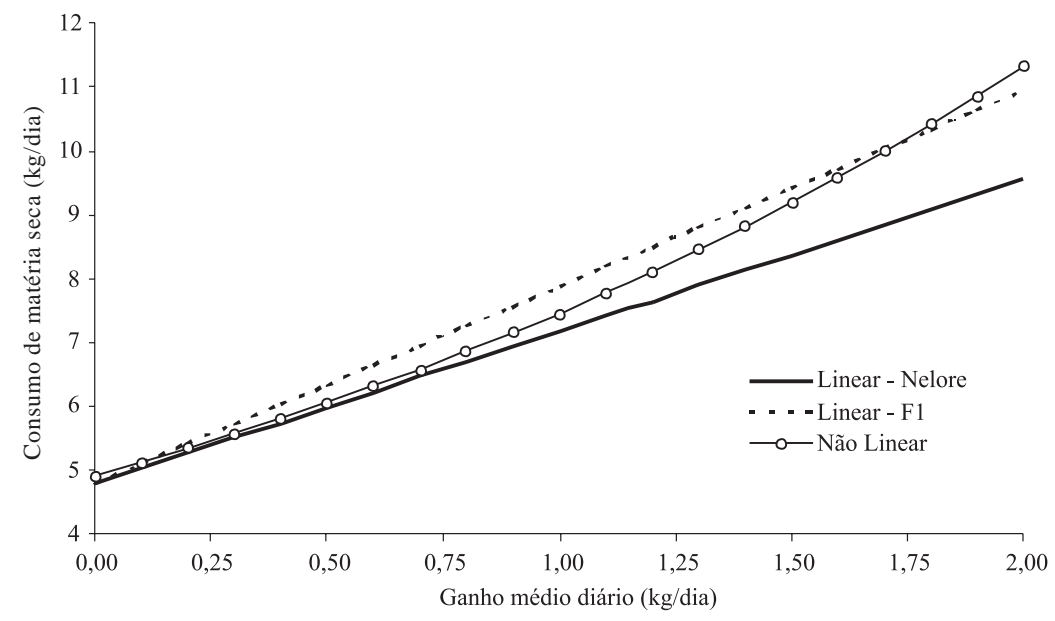

Figura 3 - Relação entre consumo de matéria seca e ganho médio diário predita pelos modelos linear (Equação 22) e não-linear (Equação 25).

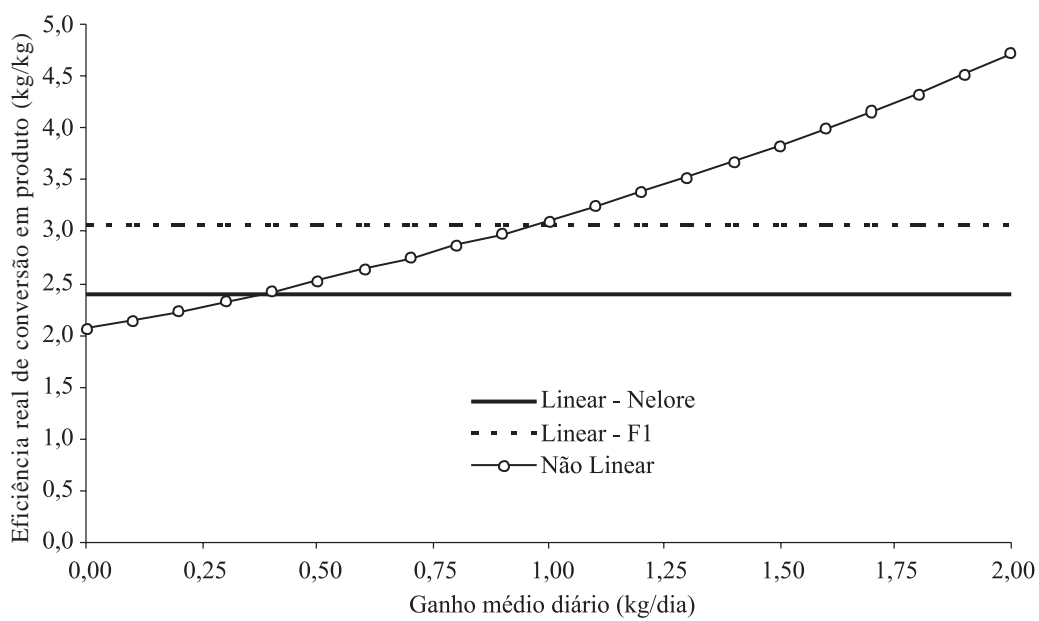

Figura 4 - Eficiências reais de conversão em produto (kg de matéria seca consumida/kg de ganho de peso) preditas pelos modelos linear (Equação 24) e não-linear (Equação 27).

apenas no contexto de demonstração do método de comparação. Interpretações nutricionais ou produtivas não devem ser retiradas deste trabalho, pois os grupos formados são heterogêneos e oriundos de diferentes condições experimentais.

Na comparação entre grupos utilizando-se a aproximação não-linear (Equações 12, 13 e 14), assumiu-se D1 = 0 e D2 = 1 para animais Nelore, e D1 = 1 e D2 = 0 para animais F1 Europeu $\times$ Nelore. Sugestão de programa utilizando-se o software SAS é apresentada na Tabela 4. Os testes de razão de máxima verossimilhança não permitiram evidenciar diferenças entre grupos $(\mathrm{P}>0,05)$ quanto aos dois parâmetros avaliados (Tabela 5). Assim, a relação entre CMS e GMD é definida conjuntamente por:

$\hat{C M S}=4,916( \pm 0,174) \times e^{0,4167( \pm 0,0303) \times G M D}\left(\mathrm{~s}_{\mathrm{XY}}=1,188\right)(25)$.

Aplicando-se o conceito de partição da eficiência sobre a equação (25), tem-se:

$$
\begin{aligned}
& D M_{N L}=\lim _{X \rightarrow 0} f(X)=4,916 \\
& E R C P_{N L}=\frac{d f(X)}{d X}=\frac{d \hat{Y}}{d X}=2,048 \times e^{0,4167 \times G M D}
\end{aligned}
$$

Tanto animais Nelore como F1 teriam DM de 4,916 kg MS/dia. Contudo, diferentemente do diagnóstico apontado pelo modelo linear (Equações 22 e 24), a ERCP não difere entre grupos (Equação 27; Figuras 3 e 4). Reitera-se que a conceituação matemática de ERCP difere nas aproximações linear e não-linear. O parâmetro ERCP estimado por modelo linear assume que, depois de supridas as exigências de mantença, a eficiência de conversão do alimento em produto é fixa e independente do nível de produção, ao passo que a aproximação não-linear assume que a ERCP varia de acordo com o nível de produção (Figura 4), havendo redução gradativa da eficiência (maior demanda de CMS por unidade de ganho) com a elevação de GMD (Detmann et al., 2011). 
A divergência quanto à diferenciação entre grupos propiciada pelos modelos linear e não-linear (Equações 22 e 25) são verossímeis, uma vez que estes diferem em sua estrutura matemática. A decisão sobre qual das aproximações (linear ou não-linear) deverá ser utilizada calca-se sobre a qualidade de ajustamento aos dados (Detmann et al., 2011).

Entre os dois modelos, aquele que apresentar a menor estimativa de AIC dever ser considerado mais adequado para descrição da relação entre CMS e GMD. Para o caso específico do conjunto de dados aqui avaliado, o modelo linear foi considerado mais adequado (Tabela 6). Logo, o conjunto de dados (Figura 2) deve ser interpretado considerando-se ERCP fixa em função do GMD, mas diferente entre grupos (Figura 3).
A utilização do AIC como critério de comparação baseia-se na sua capacidade de ponderar para o número de parâmetros do modelo (Equação 21), pois os modelos linear e não-linear ajustados poderão apresentar número diferente de parâmetros, uma vez que diferenças quanto à DM e/ou à ERCP podem ser detectadas por somente um dos modelos, como observado no exemplo apresentado neste estudo.

Apesar do embasamento matemático, estatístico e nutricional aqui demonstrados, duas limitações são observadas para avaliação da eficiência de transformação do alimento em produto por intermédio de técnicas de regressão.

Em primeiro lugar, o ajustamento dos modelos é dependente do conjunto de dados. Quanto maior a variabilidade em X (neste caso GMD), maior a confiabilidade

Tabela 4 - Programa utilizado no SAS para comparação entre grupos de acordo com o modelo não-linear

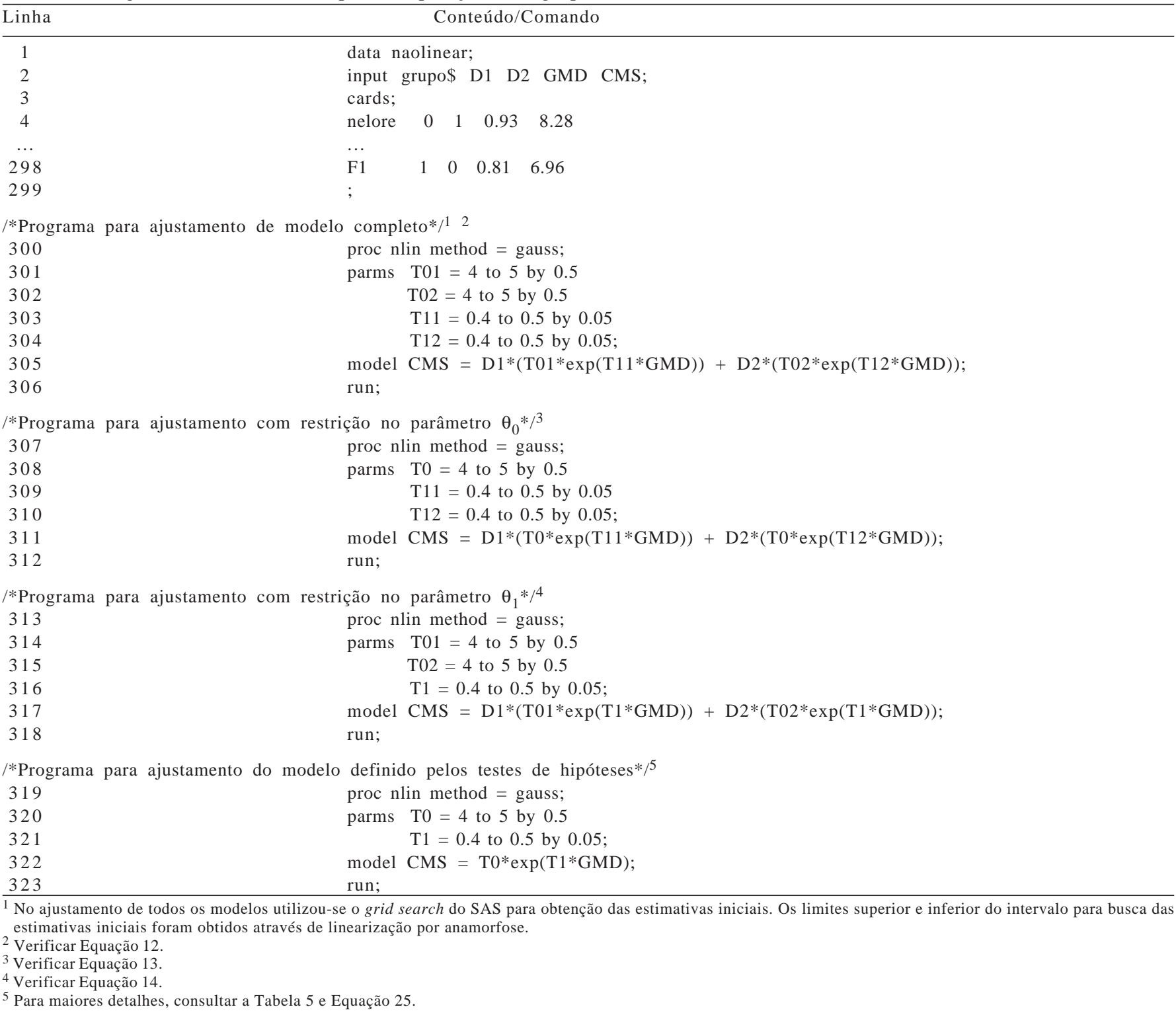


Tabela 5 - Características de avaliação do modelo não-linear (Equações 12, 13, 14 e 25)

\begin{tabular}{|c|c|c|c|c|}
\hline Modelo & Restrição/Hipótese de Nulidade & $\mathrm{p}$ & SQR & Valor-P \\
\hline Completo & - & 4 & 382,2 & - \\
\hline Restrito & $\theta_{01}=\theta_{02}=\theta_{0}$ & 3 & 382,3 & 0,7814 \\
\hline Restrito & $\theta_{11}=\theta_{12}=\theta_{1}$ & 3 & 385,2 & 0,1288 \\
\hline
\end{tabular}

$\mathrm{p}$ = número de parâmetros no modelo. $\mathrm{SQR}$ = soma de quadrados do resíduo.

Tabela 6 - Avaliação da eficiência de descrição da relação entre consumo de matéria seca e ganho médio diário pelos modelos linear (Equação 22) e não-linear (Equação 25)

\begin{tabular}{lcc}
\hline & \multicolumn{2}{c}{ Modelo } \\
\cline { 2 - 3 } Item $^{1}$ & Modelo linear & Modelo não-linear \\
\hline${ }^{\mathrm{s}_{\mathrm{XY}}} \mathrm{p}$ & 1,144 & 1,188 \\
$\mathrm{p}$ & 3 & 2 \\
$\mathrm{AIC}$ & 85,29 & 105,73 \\
\hline $\begin{array}{l}1 \mathrm{~s}_{\mathrm{XY}}=\text { desvio-padrão residual; } \mathrm{p}=\text { número de parâmetros utilizados para descrição } \\
\text { da relação; AIC = critério de informação de Akaike. }\end{array}$
\end{tabular}

do modelo ajustado (Draper \& Smith, 1966; Myers, 1990). Logo, limitações quanto às estimativas de DM e ERCP podem ser observadas em amostras de tamanho reduzido, podendo ocorrer situações em que as restrições necessárias à aplicação da técnica não sejam estabelecidas (Equações 4 e 14), inviabilizando a avaliação.

Em segundo lugar, a variável aqui proposta como variável independente (GMD) constitui variável aleatória, estando, portanto, sujeita a erro experimental. Logo, incrementos estatísticos nas estimativas de DM e ERCP e no processo de comparação entre grupos poderão ser obtidos considerando o erro inerente ao GMD no processo de estimação (Bartlett, 1949; Detmann et al., 2011).

\section{Conclusões}

A avaliação comparativa da eficiência de transformação do alimento em produto entre dois grupos experimentais de bovinos de corte por intermédio de técnicas de regressão permite, caso pertinente, a estratificação dos grupos em função da eficiência de uso da dieta para o suprimento de demandas para mantença e para produção.

\section{Referências}

AKAIKE, H. A new look at the statistical model identification. IEEE Transactions on Automatic Control, v.19, p.716-723, 1974.
BARD, Y. Nonlinear parameter estimation. New York: Academic Press, 1974. 341p.

BARTLETT, M.S. Fitting a straight line when both variables are subject to error. Biometrics, v.5, p.207-212, 1949.

DETMANN, E.; CECON, P.R.; ANDREOTTI, M.O. et al. Application of the first canonical variable in the evaluation of animal production trials. Revista Brasileira de Zootecnia, v.34, p.2417-2426, 2005.

DETMANN, E.; GIONBELLI, M.P.; VALADARES FILHO, S.C. et al. Uso de técnicas de regressão na avaliação, em bovinos de corte, da eficiência de conversão do alimento em produto: proposição de método e significância nutricional. Revista Brasileira de Zootecnia, v.40, p.2827-2834, 2011.

DRAPER, N.; SMITH, H. Applied regression analysis. New York: John Willey \& Sons, 1966. 407p.

EUCLIDES FILHO, K.; FIGUEIREDO, G.R.; EUCLIDES, V.P.B. et al. Eficiência bionutricional de animais da raça nelore e seus mestiços com caracu, angus e simental. Revista Brasileira de Zootecnia, v.31, p.331-334, 2002.

GUIDONI, A.L. Alternativas para comparar tratamentos envolvendo o desempenho nutricional animal. 1994. 105f. Tese (Doutorado em Agronomia) - Escola Superior de Agricultura "Luiz de Queiroz”/Universidade de São Paulo, Piracicaba.

KAPS, M.; LAMBERSON, W. Biostatistics for animal science. Cambridge: CAB International, 2004. 445p.

LANA, R.P.; GOES, R.H.T.B.; MOREIRA, L.M. et al. Application of Lineweaver-Burk data transformation to explain animal and plant performance as a function of nutrient supply. Livestock Production Science, v.98, p.219-224, 2005.

MYERS, R.H. Classical and modern regression with applications. Boston: PWS-Kent Publishing Co, 1990. $488 p$.

REGAZZI, A.J. Teste para verificar a igualdade de parâmetros e a identidade de modelos de regressão não-linear. Revista Ceres, v.50, p.9-26, 2003.

REGAZZI, A.J.; SILVA, C.H.O. Teste para verificar a igualdade de parâmetros e a identidade de modelos de regressão nãolinear. I. Dados no delineamento inteiramente casualizado. Revista de Matemática e Estatística, v.22, p.33-45, 2004.

REGAZZI, A.J.; SILVA, C.H.O. Teste para verificar a igualdade de parâmetros e a identidade de modelos de regressão nãolinear em dados de experimento em delineamento em blocos casualizado. Revista Ceres, v.57, p.315-320, 2010.

SOUZA, G.S. Introdução aos modelos de regressão linear e não-linear. Brasília: EMBRAPA-SPI, 1998. 505p.

VALADARES FILHO, S.C.; MARCONDES, M.I.; CHIZZOTTI, M.L. et al. (Eds.) Nutrient requirements of zebu cattle BR-CORTE. 2.ed. Viçosa, MG: DZO-UFV, 2010. 185p. 